\title{
Soft Modes, Localization, and Two-Level Systems in Spin Glasses
}

\author{
M. Baity-Jesi* \\ Departamento de Física Teórica I, Universidad Complutense, 28040 Madrid, Spain, \\ Dipartimento di Fisica, La Sapienza Università di Roma, 00185 Roma, Italy, \\ and Instituto de Biocomputación y Física de Sistemas Complejos (BIFI), 50009 Zaragoza, Spain \\ V. Martín-Mayor \\ Departamento de Física Teórica I, Universidad Complutense, 28040 Madrid, Spain, \\ and Instituto de Biocomputación y Física de Sistemas Complejos (BIFI), 50009 Zaragoza, Spain \\ G. Parisi \\ Dipartimento di Fisica, NANOTEC-CNR, Soft and Living Matter Laboratory and INFN, \\ La Sapienza Università di Roma, 00185 Roma, Italy \\ S. Perez-Gaviro \\ Centro Universitario de la Defensa, Carretera de Huesca s/n, 50090 Zaragoza, Spain, \\ and Instituto de Biocomputación y Física de Sistemas Complejos (BIFI), 50009 Zaragoza, Spain
} (Received 26 June 2015; published 23 December 2015)

\begin{abstract}
In the three-dimensional Heisenberg spin glass in a random field, we study the properties of the inherent structures that are obtained by an instantaneous cooling from infinite temperature. For a not too large field the density of states $g(\omega)$ develops localized soft plastic modes and reaches zero as $\omega^{4}$ (for large fields a gap appears). When we perturb the system adding a force along the softest mode, one reaches very similar minima of the energy, separated by small barriers, that appear to be good candidates for classical two-level systems.
\end{abstract}

DOI: 10.1103/PhysRevLett.115.267205

PACS numbers: 75.10.Nr, 75.40.Mg

Supercooled liquids and amorphous solids exhibit an excess of low-energy excitations, compared with their crystalline counterparts [1], in which at low frequencies the density of states (DOS) $g(\omega)$ has a Debye behavior $g(\omega) \propto \omega^{d-1}$ in $d$ spatial dimensions. This excess of lowfrequency modes is called a boson peak [2,3] and it is located at a small, but nonzero, frequency.

What happens at much lower frequency? Obviously, we find phonons, but what is there beyond phonons? Were it possible to disregard Goldstone bosons, a scaling $g(\omega) \propto \omega^{\delta}$, with $\delta=3$ or 4 , has been suggested for disordered systems [4,5]. Still, this has not been demonstrated nor observed. It has been stressed by Refs. [6-8] that there are localized plastic modes, whose spectral density reaches zero when $\omega$ goes to zero. These modes are subdominant in the small frequency region: They are called "plastic" because they dominate the plastic response. These extra small frequency modes may be related to the behavior of hard spheres at jamming [9-13].

Replica theory offers an explanation for these extra modes. At low enough temperatures, strongly disordered mean field models undergo spontaneous full replica symmetry breaking (RSB). Full RSB implies a complex energy landscape with a hierarchical structure of states and a large amount of degenerate minima separated by small free-energy barriers $[14,15]$. As a consequence, zero-temperature equilibrium configurations can be deformed at essentially no energy cost through easy-deformation patterns, which we name soft modes [13,16]. These modes are localized in space, but nonexponentially. In fact, the zero-temperature phase transition from the replica symmetric phase to the RSB phase is accompanied by a divergence of the localization length [17].

More often than not, finding low-lying energy minima of glassy systems is a NP problem [18]. Here, we study the behavior of inherent structures (IS), local minima of the energy obtained by relaxing the system from high temperature (the thermal protocol should not drastically change the DOS, at least if we remain in the replica symmetric phase [19]). In our study we need a model with continuous degrees of freedom. The Heisenberg model, where the spins are three-dimensional unitary vectors, is an epitome of the spin glass [26].

The global rotational symmetry of the Heisenberg spin glass has far-reaching consequences. The corresponding symmetry in structural glasses is translation symmetry (which has similar implications). The Goldstone mechanism induces soft excitations in the form of spin waves [27]. Even in disordered systems, spin waves are efficiently labeled by their wave vector, especially at low frequencies (see, e.g., Ref. [28]). As a consequence, we have a Debye spectrum $g(\omega) \propto \omega^{d-1}$, i.e., extended spin waves (for structural glasses the situation is slightly more complicated 
[29]). These symmetry-induced modes mask the physics we aim to investigate.

Thus, we add a random magnetic field (RF) to wipe out the symmetries. Indeed, mean field suggests that, if small enough, our RF does not destroy the glass phase [34]. In a RF, spin waves have a positive frequency, even for vanishing wave vector (e.g., a ferromagnet in a RF has no soft modes). Therefore, the RF exposes the (possibly localized) plastic modes that interest us. The resulting spectrum has no reason to be Debye as it does not result from plane waves. Yet, a crossover to the Debye regime should appear when the RF is small. A similar procedure of symmetry removal has been carried in glass-forming liquids, by pinning a certain fraction of particles [35-39]. The Heisenberg spin offers the advantage of allowing us to simulate unprecedentedly large systems, letting us observe scalings of several orders of magnitude.

Here, we study the ISs starting from initial random configurations and we do find that they are marginally stable states: the distribution of eigenvalues of the Hessian matrix stretches down to zero as a power law, and it is unrelated to symmetries in the system. Furthermore, we find that the soft modes are localized. We also take into account the anharmonic effects due to the complexity of the energy landscape. We find that the energy barriers along the softest mode are extremely small and that they connect very similar states with a strong relationship, which we propose as an operational definition of classical two-level systems (TLS) [40].

Model.-We study the three-dimensional Heisenberg spin glass in a RF. The dynamic variables are threedimensional spins $\vec{s}_{x}$, placed at the vertices $\boldsymbol{x}$ of a cubic lattice of linear size $L$ with unitary spacings. We have, therefore, $N=L^{3}$ spins and $2 N$ degrees of freedom due to the normalization constraint $\vec{s}_{\boldsymbol{x}}^{2}=1$. The Hamiltonian is

$$
\mathcal{H}_{\mathrm{RF}}(|\vec{s}\rangle)=-\sum_{|x-y|=1} J_{x y} \vec{s}_{\boldsymbol{x}} \cdot \vec{s}_{\boldsymbol{y}}-\sum_{\boldsymbol{x}}^{N} \vec{h}_{\boldsymbol{x}} \cdot \vec{s}_{\boldsymbol{x}},
$$

where the fields $\vec{h}_{x}$ are random vectors chosen uniformly from the sphere of radius $H_{\mathrm{amp}}$, and $|\vec{s}\rangle$ indicates the full configuration of spins $\vec{s}_{x}$. The RF breaks all rotational and translational symmetry, removing the Goldstone bosons. The couplings $J_{i j}$ are fixed, Gaussian distributed, with $\overline{J_{x y}}=0$ and $\overline{J_{x y}^{2}}=J^{2}$, where $\overline{(\cdots)}$ is the average over the disorder.

We simulated on systems of linear lattice size $L=12$, $24,48,96,192$. We chose always $J=1$, and we compared it with $H_{\text {amp }}=0.01,0.05,0.1,0.5,1,5,10,50$. In the Supplemental Material (SM) [19] we summarize the simulation parameters [19]. The case $H_{\text {amp }}=0$ will be treated in a future work [41], because it requires a different type of analysis, since the spin waves do not hybridize with the bulk of the spectrum.

Density of states.-We calculate the dynamical matrix as the Hessian matrix $\mathcal{M}$ of Hamiltonian (1), calculated at the ISs. In the SM [19] we report how the ISs were obtained, we motivate the choice of the algorithm and the temperature of the starting configuration $|\vec{s}\rangle$ for the energy minimizations, and we show how the Hessian matrix $\mathcal{M}$ was calculated.

Once $\mathcal{M}$ is known, from each simulated $H_{\text {amp }}$ we calculate the spectrum of the eigenvalues $\rho(\lambda)$ or equivalently, in analogy with plane waves [42], the DOS $g(\omega)$, by defining $\lambda=\omega^{2}$. We measure the DOS both with the method of the moments [43-45] and by explicitly computing with ARPACK [46] the lowest eigenvalues [47].

We find that, although for large fields there is a gap in the DOS, when the field is small enough the gap disappears and the DOS goes to zero, developing soft modes (Fig. 1, inset).

We focus on the $\rho(\lambda)$ for small $\lambda$, or even better on its cumulative function $F(\lambda)=\int_{0}^{\lambda} \rho\left(\lambda^{\prime}\right) d \lambda^{\prime}$. If $F(\lambda)$ reaches zero as a power law, we can define three exponents $\delta, \alpha$, and $\gamma$ that describe how the functions $g, \rho$, and $F$ go to zero for small $\lambda($ or $\omega)$ :

$$
g(\omega) \sim \omega^{\delta}, \quad \rho(\lambda) \sim \lambda^{\alpha}, \quad F(\lambda) \sim \lambda^{\gamma},
$$

where the exponents are related by $\delta=2 \alpha+1=2 \gamma-1$. In the absence of a field, one expects a Debye-like behavior $\delta=d-1=2, \alpha=0.5, \gamma=1.5$ [28].

In Fig. 1 we show the function $F(\lambda)$ for fields $H_{\text {amp }}=0.1,1$. The plots are compared with the Debye behavior $\lambda^{1.5}$ and with the power law behavior $\lambda^{2.5}$, because our data suggest a universal behavior around $\gamma=2.5$ ( $\delta=4, \alpha=1.5$ ) for all $H_{\text {amp }}$ that does not exhibit a gap [48]. See the SM [19] for the data on other $H_{\text {amp }}$ [19].

When the field is small, we remark a change of trend from $\gamma \approx 2.5$ to $\gamma<1.5$ at a value $\lambda^{*}$. Very roughly speaking, the crossover point goes as $\lambda^{*} \sim H_{\text {amp }}^{-1}$, maybe indicating the presence of a boson peak.

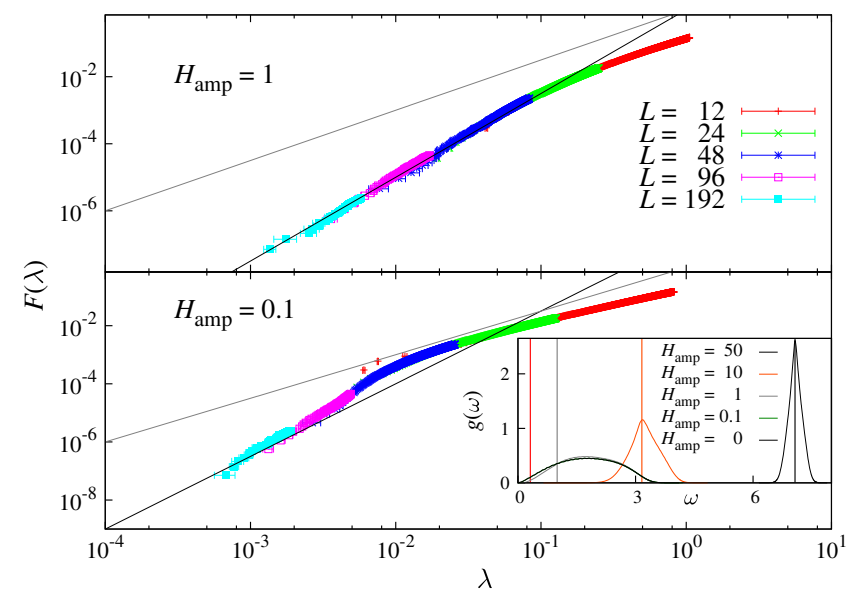

FIG. 1 (color online). Cumulative $F(\lambda)$ of the spectrum of $\mathcal{M}$. In each plot we show a black line as a reference curve representing the power law $\lambda^{2.5}$ and a gray line indicating the Debye behavior $\lambda^{1.5}$. Inset: The DOS $g(\omega)$ calculated with the method of the moments. In the limit of a diagonal Hamiltonian $(J=0)$, the DOS would be a delta function centered on $\omega^{2}=H_{\mathrm{amp}}$. This value is represented with vertical lines. 
Localization.-Similarly as it happens in other types of disordered systems [49-51], the soft modes are localized, meaning that the eigenvectors $\left|\vec{\pi}_{n}\right\rangle$ are dominated by few components. A nice localization probe is the inverse participation ratio, $Y_{n}=\sum_{x}\left(\left|\vec{\pi}_{n, x}\right|^{2}\right)^{2} /\left(\sum_{x}\left|\vec{\pi}_{n, x}\right|^{2}\right)^{2}$. If the eigenvector $\left|\pi_{n}\right\rangle$ is fully localized in one site, then $Y_{n}=1$, whereas if it is fully delocalized, $Y_{n}=1 / N$. In Fig. 2 we show that the softer the eigenvectors, the more localized they are, and for infinitely large systems there is probably a localization threshold that separates a small fixed percentage of localized eigenvectors from the delocalized bulk ones.

The localization length increases as $H_{\text {amp }}$ decreases; see Fig. 2, inset. In fact, a RSB transition should cause a localization transition at the critical $H_{\text {amp }}$ [17]. However, it is unclear whether or not a RSB transition would leave a trace in infinite-temperature ISs [52].

Anharmonicity.-We go beyond the harmonic approximation, and take into account the relationship between different ISs.

We study the reaction of the system to a force along a direction $|\pi\rangle$, normalized to one: $\sum_{x} \vec{\pi}_{x}^{2}=1$. We examine the softest mode, that is localized, and we compare it with the behavior of the eigenvectors in bulk of the $\rho(\lambda)$, that are delocalized. Therefore, we choose $|\pi\rangle=\left|\pi_{0}\right\rangle$ (softest mode) and $|\pi\rangle=\left|\pi_{\text {rand }}\right\rangle$, a vector whose components are chosen at random. The vector $\left|\pi_{\text {rand }}\right\rangle$ is not an eigenvector of $\mathcal{M}$, but it is a random linear combination of all the eigenvectors of the system. Since the bulk eigenvectors overwhelm the soft modes by number, $\left|\pi_{\text {rand }}\right\rangle$ will be representative of the bulk behavior.

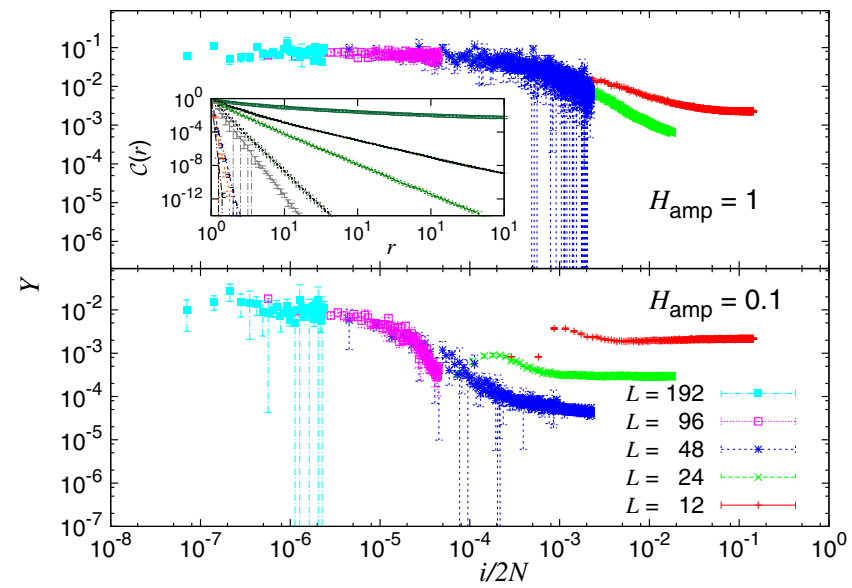

FIG. 2 (color online). Inverse participation ratio as a function of the normalized rank $i / 2 N$ of the eigenvector $(i=1$ has the smallest eigenvalue, $i=2$ the second smallest, etc.), for $H_{\text {amp }}=1$ (top) and $H_{\text {amp }}=0.1$ (bottom). Inset: Correlation function $\mathcal{C}(r)$ extracted from the eigenvectors, for fields (from top to bottom) $H_{\text {amp }}=0.01,0.05,0.1,0.5,1,5,10,50$ in $L=192$ lattices. See Ref. [19] for a close-up. For the smallest $H_{\mathrm{amp}}$, our data do not display an exponential decay, which could be caused by a localization length larger than the system size.
With the application of a forcing along $|\vec{\pi}\rangle$, Hamiltonian (1) is modified in

$\mathcal{H}_{F}(|\vec{s}\rangle)=-\sum_{\|x-y\|=1} J_{x y} \vec{s}_{x} \cdot \vec{s}_{y}-\sum_{x}^{N}\left(\vec{h}_{x}+A_{F} \vec{\pi}_{x}\right) \cdot \vec{s}_{x}$,

where $A_{F}$ is the amplitude of the forcing. If $A_{F}>0$ $\left(A_{F}<0\right)$, spins tilt toward (against) $|\vec{\pi}\rangle$. We can measure quantitatively this response of the system to the forcing through $\hat{m}=\sum_{x} \vec{s}_{x} \cdot \vec{\pi}_{x}$. We are interested in forcings $A_{F}$ both in the linear-response regime and just out of it.

We stimulate the system with forcings of increasing amplitude, and study when this kicks the system out of the original inherent structure. Ideally, the forcing amplitude $A_{F}$ would grow continuously. We simplify the analysis by choosing $A_{F}=\mathcal{A} i_{h}$, where $\mathcal{A}$ is a carefully tuned amplitude (see below), while $i_{h}$ is an integer. The unperturbed Hamiltonian corresponds to $i_{h}=0$, while $i_{h}= \pm N_{F}$ is our maximum forcing (note that $\pm i_{h}$ forcings are not equivalent due to anharmonicities).

This is how we check if new states were encountered upon increasing the forcing. (i) For each $i_{h}$, start from the IS $\left|\vec{s}^{(\mathrm{IS})}\right\rangle$ of the unperturbed Hamiltonian $\mathcal{H}_{\mathrm{RF}}$. (ii) From $\left|\vec{s}^{(\mathrm{IS})}\right\rangle$, minimize the energy using $\mathcal{H}_{F}\left(i_{h}\right)$, and find a new IS for the perturbed system, $\left|\operatorname{IS}\left(i_{h}\right)\right\rangle$. (iii) From $\left|\operatorname{IS}\left(i_{h}\right)\right\rangle$, minimize the energy again, using $\mathcal{H}_{F}(0)=\mathcal{H}_{\mathrm{RF}}$, and find the IS $\left|\mathrm{IS}^{*}\right\rangle$ (with elements $\vec{s}_{\boldsymbol{x}}^{(\mathrm{IS}) *}$ ). (iv) If $\left|\mathrm{IS}^{*}\right\rangle=\left|\vec{s}^{(\mathrm{IS})}\right\rangle$, the second minimization leads the system back to its original configuration, so the forcing was too weak to break through an energy barrier. On the contrary, if $\left|\mathrm{IS}^{*}\right\rangle \neq\left|\vec{s}^{(\mathrm{IS})}\right\rangle$, the forcing was large enough for a hop to another valley.

To ensure well-defined forcings along $\left|\vec{\pi}_{\text {rand }}\right\rangle$, we normalize $A_{F}$ with $\||\vec{\pi}\rangle \|_{1}=\sum_{x}\left|\vec{\pi}_{x}\right|$. Indeed, the perturbation in Eq. (3) is bounded by $\left|\sum_{x} \vec{\pi}_{x} \cdot \vec{s}_{x}\right| \leq\left|\sum_{x} \vec{\pi}_{x} \cdot \vec{s}_{x}\right| \leq$ $\||\vec{\pi}\rangle \|_{1}$. So, the perturbation is made extensive by choosing $A_{F}=\mathcal{A} i_{h}$, with $\mathcal{A}=N A / \||\pi\rangle \|_{1}$, where the amplitudes $A$ are an external parameter (of order 1), that we tuned in order to be in the linear-response regime for small $i_{h}$, and just out of it for $i_{h}$ approaching $N_{F}$ [19].

For the softest mode, we analyzed the effect of forcings of order $O(1)$ because larger ones lead the system out of the linear response regime, so the amplitude of the forcings along $\left|\pi_{0}\right\rangle$ is $A_{F}\left(i_{h}\right)=A i_{h} / \||\pi\rangle \|_{1}$.

See the SM [19] for further details about the linearresponse regime, hops between valleys, and the phenomenology of these rearrangements.

Two-level systems. - In the spectrum of $\mathcal{M}, \rho(\lambda)$ there is an extensive number of very soft modes, with a localized eigenstate. The eigenstates can connect different ISs through the forcing procedure we described. The connection caused by such states is privileged, because the couples of ISs are very similar one to the other. We show this in Fig. 3, where we compare the overlap $q_{\text {if }}$ between the configurations obtained through a forcing of amplitude $A_{F}\left(i_{h}\right)$ with the typical overlap between independent ISs 


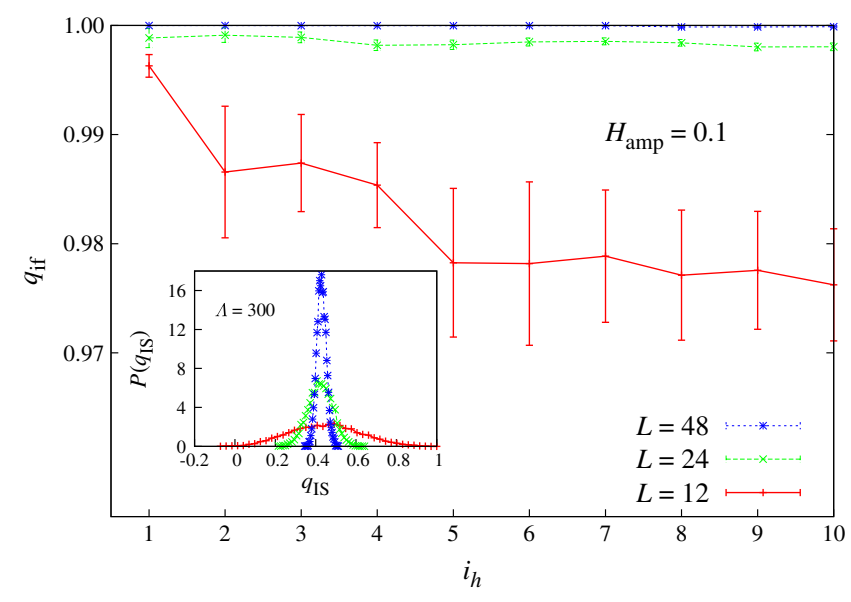

FIG. 3 (color online). Overlap $q_{\text {if }}$ between initial and forced IS (if another IS is reached) as a function of the forcing step $i_{h}$ for $H_{\text {amp }}=0.1$, for forcing along the softest mode. The inset shows the distribution of the overlaps of randomly found ISs.

[53]. This happens for every field that produces rearrangements (at $H_{\text {amp }}=10,50$ the energy landscape is too trivial and the forcings never lead to a new IS), as it can be seen from Fig. 4, top, where we show only the overlap $q_{\text {if }}$ with the largest forcings, of $i_{h}=10$. We plot $1-q_{\text {if }}$ and put it on log scale so it is better visible. The overlaps $q_{\text {if }}$ are much closer to 1 than the overlaps of independent ISs (Fig. 3, inset). This means that the ISs are somewhat clustered in tiny groups that are represented by a single IS. This could be an operational definition of classical TLS, i.e., a system in which there are two very close states, where the transitions from one state to the other can be treated as independent of the rest of the system [54-58].

Moreover, the energy barriers separating these privileged states are positive, but they do not grow with the system

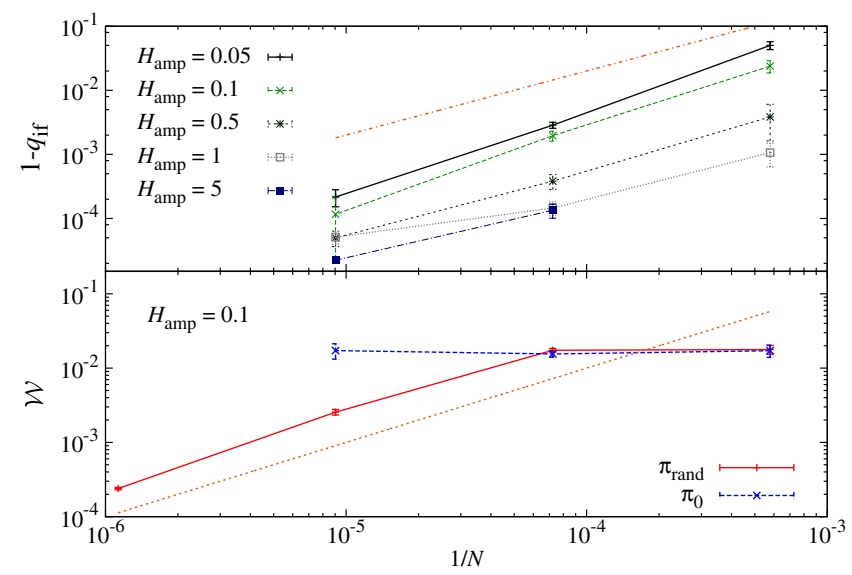

FIG. 4 (color online). Top: $1-q_{\text {if }}$ for rearrangements that occurred at the 10th forcing step, for fields $H_{\mathrm{amp}}=0.05,0.1,0.5$, 1, 5. Bottom: Cumulant $\mathcal{W}$ as a function of $1 / N$ for $\left|\vec{\pi}_{\text {rand }}\right\rangle$ and $\left|\vec{\pi}_{0}\right\rangle$. In both plots, the straight line is a reference curve $\propto 1 / N$. size (see Fig. 10 in the SM [19]). This suggests that in the thermodynamic limit $\mid$ IS $\left.^{*}\right\rangle$ and $\left|\vec{s}^{(\text {IS })}\right\rangle$ are separated by an infinitely small energy barrier, just as in a TLS.

We can get more insight on the type of rearrangement that took place during the valley change, by defining the cumulant $\mathcal{W}=\sum_{x}^{N} w_{x}^{2} /\left(\sum_{x}^{N} w_{x}\right)^{2}$, where $w_{x}=1-q_{\text {if }, x}$. If the rearrangement is completely localized, $\mathcal{W}=1$, whereas if it is maximally delocalized, $\mathcal{W}=1 / N$. Figure 4 , bottom, shows that, as we expect, the rearrangements are localized when we stimulate the system along the softest mode, and delocalized when it is along a random direction.

Conclusions.-The introduction of a random field in the Heisenberg spin glass model, besides extinguishing the rotational symmetry, qualitatively changes the shape of its DOS. Very strong random fields suppress the soft modes, and a gap appears in the DOS $g(\omega)$. Still, soft modes do resist the application of a random field when it is not too large. The data are compatible with the absence of a gap, where for small $\omega$ the DOS grows as $g(\omega) \propto \omega^{4}$, differently from the zero-field expectation, $g(\omega) \propto \omega^{2}[13,28]$.

It appears that a finite fraction of the modes is localized, suggesting a localization transition when the system becomes large.

We also analyzed the anharmonicity of the energy landscape, by imposing an external force on the system. The reaction of the spin glass has a strong dependency on the direction of application of the force. Forcings along a random direction need to be extensively strong in order to move the orientation of the spins. Equivalent results, instead, can be obtained through forcings of order 1, if they are oriented along the softest mode.

Forcings along the softest mode cause localized rearrangements that lead the system to a new IS that is infinitely similar to the original one. The two states are separated by very small energy barriers. This could be used as an operational definition of classical TLSs.

We were supported by the European Research Council under the European Unions Seventh Framework Programme (FP7/2007-2013, ERC Grant Agreement No. 247328). We were partially supported by MINECO, Spain, through the research contract No. FIS2012-35719-C02. This work was partially supported by the GDRE 224 CNRS-INdAM GREFI-MEFI. M. B.-J. was supported by the FPU program (Ministerio de Educación, Spain). The authors thankfully acknowledge the resources from the supercomputer "Memento," and the technical expertise and assistance provided by BIFI-ZCAM (Universidad de Zaragoza).

*Corresponding author. marcobaityjesi@fis.ucm.es

[1] W. A. Phillips, Amorphous Solids: Low-Temperature Properties, edited by W. A. Phillips, in Topics in Current Physics Vol. 24 (Springer-Verlag, Berlin, 1981). 
[2] U. Buchenau, N. Nücker, and A. J. Dianoux, Phys. Rev. Lett. 53, 2316 (1984).

[3] V. Malinovsky, V. Novikov, and A. Sokolov, Phys. Lett. A 153, 63 (1991).

[4] V. Gurarie and J. T. Chalker, Phys. Rev. B 68, 134207 (2003).

[5] V. L. Gurevich, D. A. Parshin, and H. R. Schober, Phys. Rev. B 67, 094203 (2003).

[6] H. G. E. Hentschel, S. Karmakar, E. Lerner, and I. Procaccia, Phys. Rev. E 83, 061101 (2011).

[7] J. Lin and M. Wyart, arXiv:1506.03639.

[8] A. L. Ning Xu, D. M. Sussman, and S. R. Nagel, Density of states for normal modes near instabilities in glasses modeled by jammed packings (to be published).

[9] M. Wyart, S. Nagel, and T. Witten, Europhys. Lett. 72, 486 (2005).

[10] M. Wyart, Phys. Rev. Lett. 109, 125502 (2012).

[11] C. S. O'Hern, L. E. Silbert, A. J. Liu, and S. R. Nagel, Phys. Rev. E 68, 011306 (2003).

[12] S. Franz, G. Parisi, P. Urbani, and F. Zamponi, arXiv:1501.03397.

[13] S. Franz, G. Parisi, P. Urbani, and F. Zamponi, Proc. Natl. Acad. Sci. U.S.A. 112, 14539 (2015).

[14] M. Mézard, G. Parisi, N. Sourlas, G. Toulouse, and M. Virasoro, Phys. Rev. Lett. 52, 1156 (1984).

[15] P. Charbonneau, J. Kurchan, G. Parisi, P. Urbani, and F. Zamponi, Nat. Commun. 5, 3725 (2014).

[16] M. Mézard, G. Parisi, and M. Virasoro, Spin-Glass Theory and Beyond (World Scientific, Singapore, 1987).

[17] C. Lupo, G. Parisi, and F. Ricci-Tersenghi (to be published).

[18] F. Barahona, J. Phys. A 15, 3241 (1982).

[19] See Supplemental Material at http://link.aps.org/ supplemental/10.1103/PhysRevLett.115.267205, which includes Refs. [20-24], for the parameters of the simulations, how to minimize the energy and a comparison between different types of IS, a derivation of the Hessian matrix, the $F(\lambda)$ for all the simulated fields, the eigenvector correlation function, a verification that the forcings were in the linear response regime, technical details on how the hops between valleys were measured, some phenomenology on the rearrangements due to the forcings, and measurements of the energy barriers in our two-level systems. For more generic data and discussions, see also Ref. [25].

[20] M. Baity-Jesi, Master's thesis, Sapienza, Universitá di Roma, 2011, arXiv:1503.08409.

[21] M. Baity-Jesi and G. Parisi, Phys. Rev. B 91, 134203 (2015).

[22] D. J. Amit and V. Martin-Mayor, Field Theory, the Renormalization Group and Critical Phenomena, 3rd ed. (World Scientific, Singapore, 2005).

[23] L. A. Fernandez, V. Martin-Mayor, S. Perez-Gaviro, A. Tarancon, and A. P. Young, Phys. Rev. B 80, 024422 (2009).

[24] M. Gell-Mann and M. Lévy, Nuovo Cimento 16, 705 (1960).

[25] M. Baity-Jesi, Ph.D. thesis, Universidad Complutense de Madrid and Sapienza Università di Roma, 2015.

[26] P. Anderson, Mater. Res. Bull. 5, 549 (1970).

[27] A. J. Bray and M. A. Moore, J. Phys. C 14, 2629 (1981).
[28] T. S. Grigera, V. Martin-Mayor, G. Parisi, P. Urbani, and P. Verrocchio, J. Stat. Mech. (2011) P02015.

[29] In the case of structural glasses, the authors of Refs. [30-33] find $g(\omega) \sim \omega^{2}$ is valid for any spatial dimension. The origin of this different behavior should be investigated carefully.

[30] M. Wyart, L. E. Silbert, S. R. Nagel, and T. A. Witten, Phys. Rev. E 72, 051306 (2005).

[31] E. Lerner, G. During, and M. Wyart, Soft Matter 9, 8252 (2013).

[32] E. DeGiuli, A. Laversanne-Finot, G. During, E. Lerner, and M. Wyart, Soft Matter 10, 5628 (2014).

[33] E. DeGiuli, E. Lerner, and M. Wyart, J. Chem. Phys. 142, 164503 (2015).

[34] A. Sharma and A. P. Young, Phys. Rev. E 81, 061115 (2010).

[35] W. Kob, S. Roldán-Vargas, and L. Berthier, Nat. Phys. 8, 164 (2012).

[36] C. Cammarota and G. Biroli, J. Chem. Phys. 138, 12A547 (2013).

[37] C. Brito, G. Parisi, and F. Zamponi, Soft Matter 9, 8540 (2013).

[38] S. Gokhale, K. Hima Nagamanasa, R. Ganapathy, and A. K. Sood, Nat. Commun. 5, 4685 (2014).

[39] K. Hima Nagamanasa, S. Gokhale, A. K. Sood, and R. Ganapathy, Nat. Phys. 11, 403 (2015).

[40] R. B. Grzonka and M. A. Moore, J. Phys. C 17, 2785 (1984).

[41] M. Baity-Jesi, V. Martín-Mayor, G. Parisi, and S. PérezGaviro (to be published).

[42] K. Huang, Statistical Mechanics, 2nd ed. (John Wiley and Sons, Hoboken, NJ, 1987).

[43] T. Chihara, An Introduction to Orthogonal Polynomials (Gordon and Breach, New York, 1978).

[44] P. Turchi, F. Ducastelle, and G. Treglia, J. Phys. C 15, 2891 (1982).

[45] J. L. Alonso, L. A. Fernández, F. Guinea, V. Laliena, and V. Martín-Mayor, Phys. Rev. B 63, 054411 (2001).

[46] D. Sorensen, R. Lehoucq, C. Yang, and K. Maschhoff, Arnoldi package (ARPACK), www.caam.rice.edu/software/ ARPACK/.

[47] The method of the moments returns the full density of states, but it is not precise at the tails. With ARPACK we can calculate exactly the smallest eigenvalues, but only a small number of them. So, when we want to show the whole spectrum, we need to use the method of the moments, while when we show the softest part of the spectrum we need ARPACK.

[48] The value $\gamma=2.5$ is also hypothesized in Ref. [4], through a fourth-order expansion of a single coordinate potential of the minimum of the energy.

[49] N. Xu, V. Vitelli, A. J. Liu, and S. R. Nagel, Europhys. Lett. 90, 56001 (2010).

[50] E. DeGiuli, E. Lerner, C. Brito, and M. Wyart, Proc. Natl. Acad. Sci. U.S.A. 111, 17054 (2014).

[51] P. Charbonneau, E. I. Corwin, G. Parisi, and F. Zamponi, Phys. Rev. Lett. 114, 125504 (2015).

[52] Inherent structures that were obtained by relaxing an infinite-temperature configuration. 
[53] The overlap $q_{\text {if }}$ between $\left|\vec{s}^{(\mathrm{IS})}\right\rangle$ and $\left|\operatorname{IS}\left(i_{h}\right)\right\rangle$ is defined as $q_{\text {if }} \equiv(1 / N) \sum_{x} q_{\text {if }, \boldsymbol{x}}$, with $q_{\text {if }, \boldsymbol{x}}=\vec{s}_{\boldsymbol{x}}^{\text {(IS) }} \cdot \vec{s}_{\boldsymbol{x}}\left(i_{h}\right)$, where $\vec{s}_{\boldsymbol{x}}\left(i_{h}\right)$ are the spins of the configuration $\left|\operatorname{IS}\left(i_{h}\right)\right\rangle$.

[54] P. W. Anderson, B. I. Halperin, and C. M. Varma, Philos. Mag. 25, 1 (1972).

[55] W. Phillips, J. Low Temp. Phys. 7, 351 (1972).
[56] W. A. Phillips, Rep. Prog. Phys. 50, 1657 (1987).

[57] J. Lisenfeld, G. Grabovskij, C. Mller, J. Cole, G. Weiss, and A. Ustinov, Nat. Commun. 6, 6182 (2015).

[58] T. Pérez-Castañeda, R. J. Jiménez-Riobóo, and M. A. Ramos, arXiv:1510.07806 [Philos. Mag. (to be published)]. 